\title{
Age differences among women in the functional asymmetry for bias in facial affect perception
}

\author{
L. S. BILLINGS, D. W. HARRISON, and J. D. ALDEN \\ Virginia Polytechnic Institute and State University, Blacksburg, Virginia
}

\begin{abstract}
The effects of age, affect valence, and cerebral asymmetry on emotional response bias and response time in the discrimination of affective facial stimuli were examined. Subjects were 11 college-aged and 11 elderly women, all of whom were right-handed. The subjects were required to identify the affect depicted in Ekman and Friesen's (1978) stimuli of angry, happy, and neutral faces by using a forced-choice reaction time paradigm with only "angry" or "happy" response alternatives signified by the respective response manipulanda. The stimuli were presented to the left and right visual fields tachistoscopically. The results showed faster affect identification by the younger women, a faster learning rate for positive affect than for negative affect, and no significantly faster identification of affect with right hemisphere (LVF) presentation. The younger and elderly women differed in the forced identification of neutral stimuli, revealing a heightened LVF positive affective bias among the elderly women. The results lend support to the hypothesis of a relative right-hemisphere decline with age.
\end{abstract}

Numerous studies of individuals with brain damage have supported the hypothesis that emotional perception is subserved to a greater extent by the right hemisphere. Bowers, Bauer, Coslett, and Heilman (1985) studied the performance of 11 right-hemisphere-damaged (RHD) subjects, 11 left-hemisphere-damaged (LHD) subjects, and 11 subjects with no evidence of past or present neurological disease (NHD) on a series of seven facial affect tasks. The performance of the RHD subjects was significantly inferior to that of the control (LHD and NHD) subjects. Dekosky, Heilman, Bowers, and Valenstein (1980) found that on a series of facial affect judgment tasks as well as a neutral facial identity task, RHD patients performed more poorly than did LHD patients.

Studies with normal subjects have also supported the hypothesis of right-hemisphere specialization for the perception of emotion (e.g., Dimond, Farrington, \& Johnson, 1976; Harrison \& Gorelczenko, 1990; Harrison, Gorelczenko, \& Cook, 1990; McKeever \& Dixon, 1981). Others have argued that both cerebral hemispheres contribute to the processing of emotion and that each hemisphere is specialized for different emotions. One hypothesis purports right-hemisphere mediation in negative affect and lefthemisphere mediation in positive affect (e.g., Sackheim \& Gur, 1978). Researchers have tested and found supporting evidence for this hypothesis through the use of unilateral hemispheric activation and reaction time paradigms.

Several researchers have studied hemispheric specialization for emotion through the use of unilateral hemispheric activation paradigms, based on Kinsbourne's (1970) model. This model provides for the assumption that acti-

Address correspondence to D. W. Harrison, Department of Psychology, College of Arts and Sciences, Virginia Tech, Blacksburg, VA 24061-0436. vation of a particular hemisphere can be achieved by focusing intention/attention in the direction opposite that hemisphere. Drake (1987) found that subjects evaluated a set of photographs more positively when the photographs were positioned in the right, as opposed to the left, visual field. Because he used essentially positive stimuli, Drake was unable to determine whether the observed differences were due to increased positivity of the left hemisphere or to increased negativity of the right. In two additional experiments, Drake (1984) demonstrated that rightward orientation produced greater personal optimism about future events than did leftward orientation. Lateral orientation was achieved by having subjects turn their bodies, heads, and eyes in the desired direction. The results of each of these studies show a greater involvement of the left hemisphere in positive affect, which implies that both the left and right hemispheres process emotional information.

A second method for studying the differential hemispheric involvement in affect involves the tachistoscopic presentation of stimuli by using a reaction time paradigm. Harrison and Gorelczenko (1990) conducted an experiment in which 50 subjects identified the facial affect depicted in Ekman and Friesen's (1978) slides of happy, angry, and neutral faces. The stimuli were presented tachistoscopically within either the right or left visual field, and the subjects' response alternatives were restricted to either "'happy" or "angry." The slowest response times were shown for "angry" stimuli presented to the right visual field (left hemisphere). The results suggested that positive affect is processed bilaterally and that negative affect is processed primarily within the right hemisphere. In similar studies, right-handed subjects have shown faster response times to pictures of faces expressing positive emotions presented to the left hemisphere and to pictures of faces expressing negative emotions presented to the 
right hemisphere (Reuter-Lorenz \& Davidson, 1981; Reuter-Lorenz, Givis, \& Moscovitch, 1983). These results suggest that positive affect is processed primarily within the left hemisphere and negative affect is processed within the right.

Taken together, the bodies of literature on unilateral hemispheric activation and tachistoscopic presentation of stimuli suggest a new methodology for studying hemispheric roles in affect perception. If the two cerebral hemispheres are, as the literature suggests, specialized for the processing of different emotions, one would expect the affective bias found by Drake (1987) (i.e., the tendency of subjects oriented to the right to rate photographs more positively than those oriented to the left) to be replicated in studies using the tachistoscopic viewing paradigm. More specifically, subjects who were asked to rate neutral faces as happy or angry would be expected to rate more often as angry the faces that were tachistoscopically presented to the left visual field, and to rate more often as happy those that were tachistoscopically presented to the right visual field.

Numerous studies have supported the hypothesis that functions of the right hemisphere decline differentially with age (Lapidot, 1987; Rastatter \& McGuire, 1990; Riege, Metter, \& Williams, 1980). A large portion of this evidence is derived from experiments that have shown visuospatial processing deficits in elderly subjects. Many studies, for example, have demonstrated a lower level of performance for elderly than for younger subjects on mental synthesis or visual integration tasks. Mason and Ganzler (1964) reported a correlation of -.45 between adult age and performance on the Hooper Visual Organization Test. Berkowitz (1953) studied the performance of subjects of different ages on several subtests of the Wechsler Adult Intelligence Scale. The most marked declines in performance with increasing age were found on the digit symbol, block design, picture arrangement, picture completion, and object assembly subtests. The other subtests used in the study were verbal. These results suggest that the right hemisphere (e.g., visuospatial processing) declines more rapidly with age.

Attenuation of emotional processing skills in the elderly also supports the hypothesis that right hemispheric functions decline with age. McDowell and Harrison (1993) found age differences in the accuracy of facial emotion identification. In that study, subjects identified five affect categories presented in a photo album. The elderly and younger participants were found to have equivalent accuracy scores for happy faces. Significant declines in accuracy scores with age were seen across each negative affective valence (sadness, anger, fear). As noted in Moreno, Borod, Welkowitz, and Alpert (1990), research has shown that individuals tend to become more introverted, more cautious, and less emotionally responsive to the external environment with advancing age (Botwinick, 1973; Rosen \& Neugarten, 1960). In a study conducted by Malatesta, Izard, Culver, and Nicolich (1987), young, middle-aged, and elderly women viewed videotapes of facial expressions and rated the expressions for emotional intensity as well as for the type of affect being communicated. Overall, the elderly subjects performed more poorly on this task. A response bias was also observed whereby the elderly subjects attributed more interest and contempt to faces than did the middle-aged group, and the middle-aged subjects attributed more anger to faces than did the elderly subjects. Although the researchers did not discuss their findings from a neuropsychological perspective, their results nonetheless seem to suggest a decline in the processing of negative affect in the elderly. If emotion, particularly negative emotion, is processed by the right hemisphere, both the inferior performance of the elderly subjects on this emotional decoding task and their tendency to rate faces as interested or contemptuous more often than as angry (a stronger negative affect) support the hypothesis of a righthemisphere decline with age.

The purpose of the present study, in which women subjects identified the affect of slides presented tachistoscopically to either the right or left visual field, was to examine the effects of age on hemispheric asymmetry in response bias and on the identification of affective facial stimuli. In one part of the experiment, the subjects were tested on their perception of neutral faces as being either happy or angry. On the basis of recent research supporting affective bias (Drake, 1987) and right-hemisphere decline with advancing age (Rastatter \& McGuire, 1990), it was hypothesized that the elderly women would identify neutral facial stimuli presented to the left visual field as happy more often than would the younger women. Reduced asymmetry among the elderly was predicted to result from right-hemisphere aging. Second, it was hypothesized that the affective stimuli would be processed more rapidly when presented to the left visual field, thereby supporting the hypothesis that emotion in general is perceptually subserved to a greater extent by the right hemisphere. This left-visual-field advantage was expected to be minimal, however, due to the somewhat symmetrical hemispheric representation of emotional skills in women (Harrison \& Gorelczenko, 1990). Third, on the basis of Harrison and Gorelczenko's study, it was hypothesized that the subjects would learn to identify positive affect more quickly than negative affect. Finally, it was hypothesized that the elderly women would process emotional information at significantly slower rates than the younger women. This result was expected because reaction time has been shown to increase with generalized decline due to age (e.g., Salthouse, 1979).

\section{METHOD}

Subjects

The subjects were 11 female college students with a mean age of 19.17 $(S D=0.94)$ and 11 elderly women with a mean age of $72.91(S D=$ 6.07). The younger subjects were volunteers from the introductory psychology pool of Virginia Polytechnic Institute and State University. The elderly subjects were recruited from among the residents of a retirement community in Blacksburg, VA. The education of the residents ranged from completion of high school to four years in college. Their parents' average educational level was junior high school. The residents, who had been retired for an average of 7-10 years, earned an average 
annual income of about $\$ 25,000$ and had lived at their current address for an average of 1-3 years. Informed consent from all the subjects as well as Institutional Review Board approval were obtained prior to the study.

\section{Handedness and Visual Acuity}

The handedness of the subjects was determined through the administration of a 13-item behaviorally validated questionnaire (Coran, Porac, \& Duncan, 1979), designed to assess four types of lateral preference (hand, foot, eye, and ear). The questionnaire was developed from several preexisting laterality inventories and behavioral tests for types of lateral preference. The average concordance between behavioral and self-report measures was $\mathbf{9 0}$. Self-report items included in the questionnaire were scored as +1 for "right," -1 for "left," and 0 for "both" (either right or left). Possible scores varied between - 13 (strong left-handedness) and +13 (strong right-handedness), inclusively. The criterion for right-hand dominance and inclusion in the study was a minimum total score of +6 . Each subject also read a Snellen eye chart at a distance of $20 \mathrm{ft}$ to measure visual acuity. The criterion for inclusion in the study was corrected vision of $20 / 40$ or better.

\section{Stimuli and Apparatus}

The stimuli consisted of slides produced from 30 pictures of facial affect (10 happy, 10 angry, and 10 neutral), which were randomly selected from the collection developed by Ekman and Friesen (1978). The photographs were remounted so that the stimulus face appeared in either the right (RVF) or left (LVF) visual half-field. The inside edge of the picture was positioned $3^{\circ}$ from the center, and the outside edge was positioned $12^{\circ}$ from the center. These procedures resulted in a total of 60 slides (10 RVF happy, 10 RVF angry, 10 RVF neutral, 10 LVF happy, 10 LVF angry, and 10 LVF neutral).

A constant-illumination tachistoscope was used to project the stimuli onto a screen, which was positioned $2.67 \mathrm{~m}$ in front of the subject. The center of the screen was marked with a black dot positioned $1.47 \mathrm{~m}$ above the floor. In the sound-attenuated experimental chamber, the luminance level was $4.5 \mathrm{~cd} / \mathrm{m}^{2}$ and was reduced to $2.5 \mathrm{~cd} / \mathrm{m}^{2}$ during the presentation of a slide. The experimenter and automated programming equipment were located in a room separate from the subject, who was monitored through an observation window and prompted by means of an intercom. Trial onset was signaled by a $2000-\mathrm{Hz}, 55-\mathrm{dBA}$ tone that was sounded behind the subject. The manipulanda consisted of two "soft touch" trip switches flush-mounted on the midline of a right-handed student desk. The switches, separately labeled "happy" and "angry," were positioned $58.5 \mathrm{~cm}$ from the back of the chair.

\section{Procedure}

Each subject was administered the vision test, handedness questionnaire, and the following instructions:

In this part of the study you will have to make decisions concerning faces which you will see on the screen. The presentation of faces will be brief and either to the left or to the right of the black dot. The presentation of the face will be preceded by a tone [the tone is sounded]. We ask that upon hearing the tone you focus on the black dot because the face will be presented about three seconds after the tone. We also ask that you use your right index finger to choose whether the face is happy or angry. Please keep your index finger raised above and between the two switches. After the presentation of the face, please make your selection by pressing the switch labelled "happy" or "angry." Please respond as quickly and as accurately as possible. To get you used to the procedure, practice trials will be provided. Also, we will inform you when the practice trials end and the study begins. There is an intercom located behind you if you need to contact us. We will remind you to fixate on the black dot during the testing. Any questions?

After having read the instructions, the subject was shown a series of 20 practice slides. The presentation of a slide was signaled by a $1-\mathrm{sec}$ tone; $3 \mathrm{sec}$ after the tone, the stimulus was shown for $250 \mathrm{msec}$. The subject identified the affective valence of the face through the use of a two-choice reaction time paradigm with an intertrial interval of $15 \mathrm{sec}$. Each subject's inclusion in the study was contingent upon her correct identification of eight consecutive slides within the first three replications of the practice slides.

During the test phase, the slide set consisted of 60 slides, each of a face portraying one of three affects: happy, angry, or neutral. The subject was periodically reminded to focus on the fixation point in the center of the screen to ensure the integrity of stimulus presentation within either visual field. To eliminate position effects, the location of the response keys, corresponding to "positive" or "negative" affect, were counterbalanced across subjects. Order effects were controlled through the use of three randomized orders of slide presentation.

\section{RESULTS}

Separate mixed-design analyses of variance (ANOVAs) were performed on the reaction time and the response bias data. The ANOVA on the response bias data (neutral faces were identified as angry or happy) consisted of a twofactor mixed-design ANOVA with an independent factor of age and repeated measures of visual field. This ANOVA revealed a significant interaction effect only for age $\times$ visual field $[F(1,20)=4.38, p<.05]$.

As Figure 1 indicates, both the younger and elderly women chose the "angry" response alternative at approximately equal rates when the neutral stimuli were presented to the right visual field. When the neutrally toned faces were presented to the left visual field, however, the younger women were more likely than were the elderly women to identify them as angry.

The ANOVA on the reaction time data (to angry and happy faces) consisted of a four-factor mixed design with age as an independent factor and with repeated measures on visual field, slide affect (positive and negative), and trial (Trials 1-10).

No significant main effect of visual field was observed. The mean reaction time for slides presented to the left visual field was $1.33 \mathrm{sec}$, and the mean reaction time for slides presented to the right visual field was $1.43 \mathrm{sec}$.

Reaction time varied significantly as a function of slide affect and trial. The slide affect $\times$ trial interaction was significant $[F(9,180)=1.98, p<.05]$. The decrease in reaction time was reliably greater for happy than for angry faces across trials. The main effect for slide affect was significant $[F(1,20)=7.31, p<.02]$. The mean reac-

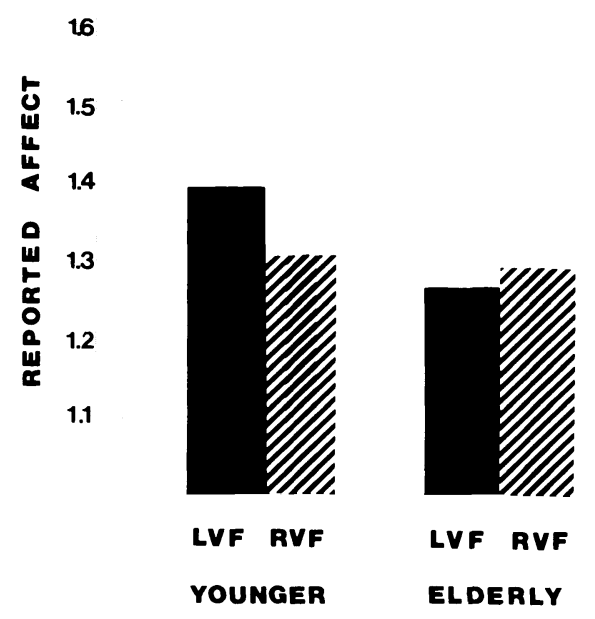

Figure 1. Mean reported affect for the neutral faces ( 1 = happy; 2 = angry) as a function of visual field. 
tion times for happy and angry faces were 1.27 and $1.49 \mathrm{sec}$, respectively.

The reaction time analysis revealed a significant main effect of age $[F(1,20)=4.31, p<.05]$, indicating that the younger subjects processed affective information more quickly than did the elderly subjects. The mean response times for the younger and elderly subjects were 1.23 and $1.53 \mathrm{sec}$, respectively.

\section{DISCUSSION}

The present study yielded four primary findings. Most important, the subjects demonstrated age differences in affective bias in the identification of neutral stimuli restricted to presentation of neutral faces to the right hemisphere. The faces were identified as angry less often when they were presented to the right hemisphere of the elderly women. The younger women were more likely to identify as angry the faces presented to the left visual field. This asymmetry in response bias was not present among the elderly group; the elderly women were less likely to identify as angry the faces presented to the left visual field. These results support the hypotheses that the right hemisphere is specialized for the processing of negative emotion and that right-hemisphere affective response bias declines with age.

It is interesting to note that these findings are inconsistent with those obtained by Drake (1987). In studying the effects of gaze manipulation, Drake found that women subjects demonstrated no affective bias. The presence of affective bias in the present study could be due to the small sample size $(N=22)$. Alternatively, the differences in the results of these two studies may be due to methodological differences. Drake used only positive stimuli, and subjects turned their heads, bodies, and eyes to the left or right in order to view the stimuli. In the present study, we used a forced-choice paradigm with neutral stimuli and the subjects were required to focus on a central fixation point while the stimuli were presented to either the left or right visual field.

Interestingly, the age $\times$ visual field interaction was not significant in the analysis of the reaction time data. This may be due to the confounding of peripheral performance variables associated with the aging process. The elderly women exhibited overall slower reaction times with heightened variability in performance. Due to increased variability in performance and slower response times among the elderly in general, using reaction time measures may obscure age-related central nervous system differences in the identification of facial affect.

The results revealed no significant main effect of visual field. However, the mean reaction time for the identification of angry and happy faces was slightly faster for the left visual field. This result is consistent with research reporting reduced lateralization for women on this task (Harrison \& Gorelczenko, 1990). However, this result has a plausible alternative explanation. If the right hemisphere is, in fact, especially susceptible to the aging process, then elderly women should evidence overall slower reaction times for the identification of stimuli presented to the left visual field. The fact that the reaction time data did not yield a significant left-visual-field advantage could be due to the combination of the reaction times of younger and elderly women.

The happy faces were identified more rapidly than the angry faces, irrespective of visual field. For each subject, reaction times were recorded across 10 trials for each affect (positive and negative). In general, reaction time decreased across the 10 trials, irrespective of affect. This decrement, a measure of learning, was reliably greater for positive affect. The results indicate that, by using these procedures, the identification of positive affect is learned faster than is the identification of negative affect.

Finally, the results indicate that the elderly women processed happy and angry faces at significantly slower rates than did the younger women. This is consistent with research reporting a general increase in reaction time with age (e.g., Salthouse, 1979).

\section{REFERENCES}

Berkowitz, B. (1953). The Wechsler-Bellevue performance of white males past age 50. Journal of Gerontology, 8, 76-80.
Botwinick, J. (1973). Aging and behavior. New York: Springer. Bowers, D., Bauer, R. M., Coslett, H. B., \& Heilman, K. M. (1985). Processing of faces by patients with unilateral hemisphere lesions: 1. Dissociation between judgments of facial affect and facial identity. Brain \& Cognition, 4, 258-272.

Coran, S., Porac, C., \& Duncan, P. (1979). A behaviorally validated self-report inventory to assess four types of lateral preference. Journal of Clinical Neuropsychology, 1, 55-64.

Dekosky, S., Heilman, K., Bowers, D., \& Valenstein, E. (1980). Recognition and discrimination of emotional faces and scenes. Brain \& Language, 9, 206-218.

Dimond, S. J., FARRINGTON, L., \& Johnson, P. (1976). Differing emotional responses from right and left hemispheres. Nature, 261, 690-692.

DRAKE, R. A. (1984). Lateral asymmetry of personal optimism. Journal of Research in Personality, 18, 497-507.

DRAKE, R. A. (1987). Effects of gaze manipulation on aesthetic judgments: Hemisphere priming of affect. Acta Psychologica, 65, 91-99.

Ekman, P., \& Friesen, W. (1978). Pictures of facial affect. Palo Alto, CA: Consulting Psychologist Press.

Harrison, D. W., \& Gorelczenko, P. M. (1990). Functional asymmetry for facial affect perception in high- and low-hostile men and women. International Journal of Neuroscience, 55, 89-97.

Harrison, D. W., Gorelczenko, P. M., \& CoOK, J. (1990). Sex differences in the functional asymmetry for facial affect perception. International Journal of Neuroscience, 52, 11-16.

KinsBourne, M. (1970). The cerebral basis of lateral asymmetries in attention. Acta Psychologica, 33, 193-201.

LAPIDOT, M. (1987). Does the brain age uniformly? Evidence from effects of smooth pursuit eye movements on verbal and visual tasks. Journal of Gerontology, 42, 329-331.

Malatesta, C. Z., Izard, C. E., Culver, C., \& Nicolich, M. (1987). Emotion communication skills in young, middle-aged, and older women. Psychology \& Aging, 2, 193-203.

Mason, C. F., \& Ganzler, H. (1964). Adult norms for the Shipley Institute of Living Scale and Hooper Visual Organization Test based on age and education. Journal of Gerontology, 19, 419-424.

MCDOWELL, C. L., \& HARRISON, D. W. (1993). Age differences in negatively valenced but not positive valenced emotional face perception. Manuscript submitted for publication.

MCKeEver, W. F., \& Dixon, M. S. (1981). Right-hemisphere superiority for discriminating memorized from nonmemorized faces: Affective imagery, sex, and perceived emotionality effects. Brain \& Language, 12, 246-260.

Moreno, C. R., Borod, J. C., Welkowitz, J., \& Alpert, M. (1990). Lateralization for the expression and perception of facial emotion as a function of age. Neuropsychologia, 28, 199-209.

Rastatter, M. P., \& McGuire, R. A. (1990). Some effects of advanced aging on the visual-language processing capacity of the left and right hemispheres: Evidence from unilateral tachistoscopic viewing. Journal of Speech \& Hearing Research, 33, 134-140.

Reuter-Lorenz, P., \& Davidson, R. J. (1981). Differential contributions of the two cerebral hemispheres to the perception of happy and sad faces. Neuropsychologia, 19, 609-613.

Reuter-Lorenz, P. A., Givis, R. P., \& Moscovitch, M. (1983). Hemispheric specialization and the perception of emotion: Evidence from right-handers and from inverted and noninverted left-handers. Neuropsychologia, 21, 687-692.

Riege, W. H., Metter, E. J., \& Williams, M. V. (1980). Age and hemispheric asymmetry in nonverbal tactile memory. Neuropsychologia, 18, 707-710.

Rosen, J. L., \& Neugarten, B. (1960). Ego functions in the middle and later years: A thematic apperception study of normal adults. Journal of Gerontology, 15, 62-67.

SACKHEIM, H., \& GUR, R. C. (1978). Lateral asymmetry in intensity of emotional expression. Neuropsychologia, 16, 473-481.

Salthouse, T. A. (1979). Adult age and the speed-accuracy trade-off. Ergonomics, 22, 811-821. 